außerdem von der Korngröße abhängig gewesen sein. Man muß auch die Möglichkeit erwägen, daß Urgase ebenso wie radiogenes Argon durch Diffusion verlorengegangen sind.

Die Proportionalität zwischen Urgasgehalt und $\mathrm{Xe}^{129}$. Abweichung gilt offenbar nicht für die Fraktionen, während sie für die ungetrennten Proben ${ }^{1}$ angenähert gilt. Demnach wurden die Urgase auf andere Weise einge- baut als $\mathrm{Xe}^{129}$. Möglicherweise entstand $\mathrm{Xe}^{129}$ teilweise durch Teilchenstrahlung. Dann müßten die Chondren stärker bestrahlt worden sein als Matrix und Eisen. Da die Chondren weniger Urgase besitzen, kann man vermuten, daß sie auch höher erhitzt wurden als die Matrix.

Für eine Entscheidung sind jedoch noch weitere Untersuchungen nötig.

\section{Eine Mikrowellen-Meßmethode zum Nachweis intensiver Stoßwellen in nichtleitenden Festkörpern}

\author{
H. D. vom Stein, B. Koch und R. Schall
}

Deutsch-FranzösischesForschungsinstitut St. Louis (Ht. Rhin), Frankreich

(Z. Naturforschg. 20 a, 157-158 [1965]; eingeg. am 7. Dezember 1964)

Im Verlauf von Messungen der Detonationsgeschwindigkeit in festen Sprengstoffen mit Hilfe eines Mikrowellen-Reflexions-Interferometers ${ }^{1}$ wurde im Anschluß an das Signal, das von der mit konstanter Geschwindigkeit durch den Sprengkörper fortschreitenden Detonationsfront herrührt, ein Signal beobachtet, das einem mit abnehmender Geschwindigkeit verlaufenden Vorgang entspricht und daher zweifelsohne der Stoßwelle zuzuordnen ist, die in dem an den Sprengkörper angrenzenden Isolationsmaterial erzeugt wird.

Während die Reflexion der Mikrowellenstrahlung an der Detonationsfront im wesentlichen durch deren starke Ionisation ${ }^{2}\left(n_{\mathrm{e}} \approx 10^{19} \mathrm{~cm}^{-3}\right)$ bewirkt wird, ist anzunehmen, daß die Reflexion an der Stoßfront in kompakten Isolierstoffen auf den Dichtesprung zurückzuführen ist, der eine Unstetigkeit in der DK darstellt.

Die Meßmethode beruht auf einer bereits früher mehrfach benutzten Anordnung ${ }^{3-5}$ (Abb. 1) .

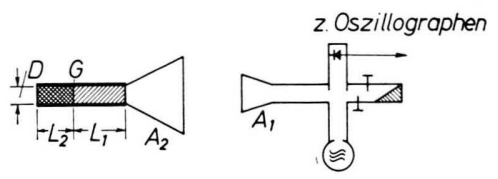

Abb. 1. Schema der Meßanordnung. $L_{2}$ Sprengstoffstrecke $=60 \mathrm{~mm}, L_{1}$ Isoliermaterial $=80 \mathrm{~mm}, D=10 \mathrm{~mm}$. $f_{0}=34,5 \mathrm{GHz} ; \lambda_{0}=8,69 \mathrm{~mm}$.

Ein Metallzylinder, der jeweils zur Hälfte mit Sprengstoff (gegossenem TNT/Hexogen) und dem untersuchten Material (Sand bzw. Polystyrol) gefüllt ist, dient als Wellenleiter der von der Antenne $A_{1}$ über einen aus Aluminiumfolie hergestellten Trichter $A_{2}$ eingespeisten Mikrowelle ${ }^{6}$.

1 Über diese wird an anderer Stelle berichtet.

2 M. Birk, A. Erez, Y. Manheimer u. G. Nahmani, Bull. Res. Council Israel, Sect. A 3, 4 [1954].

3 B. Косн, C. R. Acad. Sci., Paris 236, 661 [1953].

4 G. F. Cawsey, J. L. Farrands u. I. Thomas, Proc. Roy. Soc., Lond. A 248, 499 [1958].
Bei der Zündung des Sprengstoffes von der linken Seite der Zeichnung her bildet sich eine hochionisierte Front aus, die mit Detonationsgeschwindigkeit von links nach rechts durch den Sprengstoff läuft und an der die Mikrowelle quasimetallisch reflektiert wird. Die durch den Doppler-Effekt bedingte Frequenzverschiebung $f_{\mathrm{D}}$ der reflektierten Welle wird registriert; sie ergibt gemäß der Beziehung

$$
v=f_{\mathrm{D}} \cdot \lambda / 2
$$

die Geschwindigkeit $v$ der Detonationsfront im Sprengstoff. Dabei ist $\lambda$ die unmittelbar vor der Detonationsfront vorhandene Wellenlänge.

Da jede Doppler-Schwebung einer Verschiebung der Reflexionsebene um $\lambda / 2$ entspricht, erhält man aus der Zahl der Doppler-Schwebungen bei Kenntnis von $\lambda$ die räumliche Verschiebung der Reflexionsfront als Funktion der Zeit. Es ergeben sich Registrierungen nach Art der in Abb. 2 a, b * dargestellten Beispiele, in denen auf den mit einer Überdeckung von etwa $20 \%$ der Kippdauer der oberen Zeitbasis aneinander anschließenden Zeitlinien eines Zweistrahl-Oszillographen das DopplerOszillogramm des Vorganges im Bereich um die in Abb. 1 mit $G$ bezeichnete Kontaktfläche zwischen Sprengstoff und Festkörper mit verschiedener Zeitauflösung dargestellt ist. Der Zeitpunkt, an dem diese Grenze von der Detonationswelle überlaufen wird, ist aus dem Zeitabstand zwischen der Zündung des Sprengkörpers und der Triggerung des Oszillographen bekannt (Zeitmarke $4 \mathrm{MHz}$ ).

Die quantitative Auswertung der Oszillogramme ergiht für den Anfangsteil der Registrierung zunächst die stationäre Geschwindigkeit der Detonationsfront von $7300 \mathrm{~m} / \mathrm{s} \mathrm{bzw} .7600 \mathrm{~m} / \mathrm{s}$ bis an die Grenze G. Aus der Übereinstimmung dieser Zahlen mit den aus anderen Messungen bekannten Werten ist gleichzeitig ein Rückschluß auf die vorzugsweise Anregung der Hauptwelle $\left(\mathrm{H}_{11}\right)$ in dem an sich übermodigen Hohl. leiter möglich. Für den benutzten Sprengstoff wurde eine ebenfalls bekannte DK $\varepsilon_{\mathrm{r}}=3,42$ angenommen, entsprechend einer Wellenlänge $\lambda=4,85 \mathrm{~mm}$.

5 B. Koch, H. Freiwald u. H. W. Koch, Z. Naturforschg. 19 a, 396 [1964].

${ }^{6}$ Die Luftstrecke ist lediglich zum Schutz der Mikrowellenapparatur vorgesehen.

* Abb. 2 a, b siehe S. 156 a. 


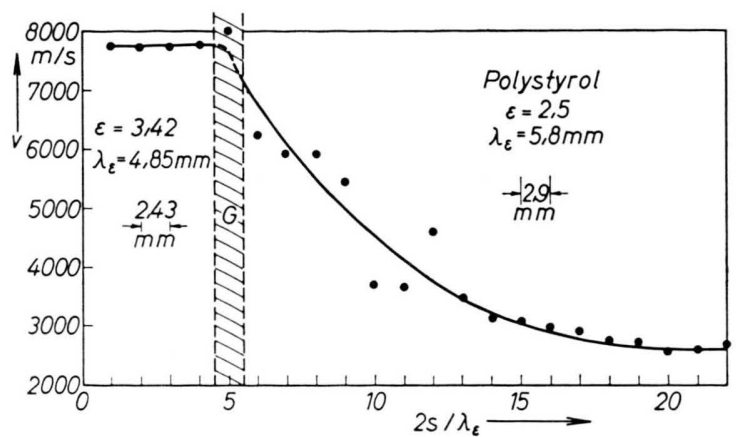

Abb. 3 a. Geschwindigkeitsverlauf beim Übergang der Detonations-Stoßwelle in Polystyrol bei G. Ordinate: Geschwindigkeit; Abszisse: relative Wegstrecke $2 s / \lambda_{\varepsilon}$ (wahre Wegstrecken zwischen zwei Meßpunkten innerhalb des Diagramms angegeben). Schraffierter Bereich: Ubergangszone mit unsicherer „wirksamer“ DK.

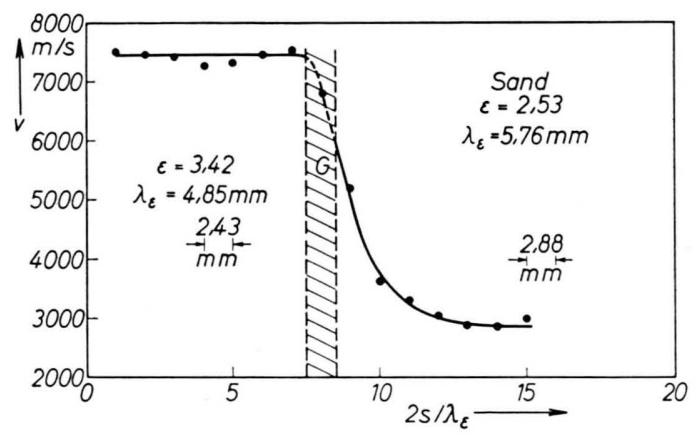

Abb. 3 b. Geschwindigkeitsverlauf beim Utbergang der Detonations-Stoßwelle in vorgepreßten Sand. Sonst wie in Abb. 3a.

Aus dem nach Überschreiten des Punktes $G$ weiterhin auswertbaren (wenn auch weniger regelmäßigen)

7 R. F. Harrington, Time-harmonic Electromagnetic Fields, McGraw-Hill Book Co., New York 1961, S. 454.
Doppler-Signal ergeben sich unter Berücksichtigung der DK-Werte für Polystyrol $\left(\varepsilon_{\mathrm{r}}=2,5\right)$ und trockenem Sand $^{7} \quad\left(\varepsilon_{\mathrm{r}}=2,53\right) \quad$ Geschwindigkeitskurven nach Abb. $3 \mathrm{a}$, b. Dabei besteht allerdings im Übergangsgebiet um $\mathrm{G}$ eine gewisse Unsicherheit über das „wirksame“ $\varepsilon_{\mathrm{r}}$, da sich hier der DK-Sprung auf wenigstens einen Meßabschnitt $(\lambda / 2)$ auswirkt, wozu möglicherweise noch der Einfluß von Feldverzerrungen tritt (vgl. das Registrierbeispiel der Abb. 2 a). Infolgedessen muß ohne nähere Kenntnis dieser Störungen den in diesem Übergangsgebiet liegenden ein oder zwei Meßpunkten ein geringeres Gewicht beigemessen werden als den restlichen Meßwerten.

Offensichtlich ist der beobachtete Geschwindigkeitsverlauf der in den Festkörper auslaufenden Stoßwelle zuzuordnen. Während beim porösen Sand die verhältnismäßig starke Reflexion der Mikrowellenenergie an der Stoßfront noch durch einen Beitrag der Ionisierung der in den Poren eingeschlossenen Luft erklärt werden könnte, muß für das kompakte Polystyrol angenommen werden, daß der in der Stoßfront vorliegende Dichte- und der mit ihm verbundene DK-Sprung für die Reflexion verantwortlich ist, wie dies ähnlich auch für nichtionisierte schwache Luftstoßwellen nachgewiesen wurde ${ }^{8}$.

Da bei den vorliegenden Versuchen der Amplitudenverlauf des Doppler-Signals merklich durch die Filtercharakteristik des Meßverstärkers mitbedingt ist, erlauben diese Messungen noch keine genaueren Angaben über das Reflexionsvermögen der sich im Festkörper ausbreitenden Stoßfront. Die Festlegung eines quantitativen Zusammenhanges zwischen der Geschwindigkeit der Stoßfront, dem dort vorliegenden Dichtesprung und dem Mikrowellen-Reflexionsvermögen würde eine wertvolle Ergänzung zur Methode der Untersuchung von Stoßwellen-Vorgängen in Festkörpern mit Hilfe von Röntgen-Blitzen ${ }^{9}$ darstellen. Derartige Untersuchungen sind zur Zeit im Gange.

8 B. Косн, Phys. Verh. 8, 191 [1957] ; Z. Naturforschg. 19 a, 395 [1964].

9 R. Schall, Explosivstoffe 6, 120 [1958].

\section{Zum Plasma-Mikrofeld}

\section{K. Hunger, R. W. Larenz und K. Wilke \\ Institut für Theoretische Physik der} Technischen Hochschule Hannover

(Z. Naturforschg. 20 a, 158-159 [1965] ; eingeg. am 14. Dezember 1964)

Zur Prüfung bestehender Mikrofeldtheorien wurde folgendes numerische Experiment mit Hilfe der elektronischen Rechenanlage CDC 1604 A der T. H. Hannover durchgeführt: Ein aus Ionen und Elektronen bestehendes ideales Gas mit insgesamt $N$ Plasmateilchen wurde in einem Kubus eingeschlossen und dann sich selbst überlassen. Hierbei bewegen sich die Teilchen von beliebig wählbaren Anfangslagen mit beliebig vorgebbarer Geschwindigkeitsverteilung auf gradlinigen Bahnen zwischen den Wänden des Kubus (streng genommen zwischen „Reflexionsschichten“, die infolge endlicher Bahnintegrationsschrittweite eine endliche Schichtdicke besitzen; wegen der Einzelheiten des Rechenverfahrens sei auf künftige Veröffentlichungen verwiesen). Im Inneren des Kubus kann an einem Aufpunkt das von den Plasmateilchen erzeugte Mikrofeld, das Potential etc. in jedem Zeitpunkt registriert und bezüglich seiner Wahrscheinlichkeitsverteilung von der Rechenmaschine festgehalten werden. Für Teilchenzahlen der Ordnung $N \leqq 10^{2}$ ergab sich, abgesehen vom Bereich hoher Feldstärken, die bekannte Holtsmark- 
Mikrofeldverteilung ${ }^{1}$ (von $N=\infty$ auf die endliche Zahl $N$ unwesentlich korrigiert). Dagegen ergaben sich für $N \geqq 10^{3}$ deutliche Abweichungen hiervon (s. Abb. 1), und zwar im Sinne größerer mittlerer Feldstärken, wie dies in Arbeiten der oben an 1. und 2. Stelle genannten Autoren ${ }^{2,3}$ für Temperaturen $k T>e^{2} n^{1 / 3}$ (Debye-

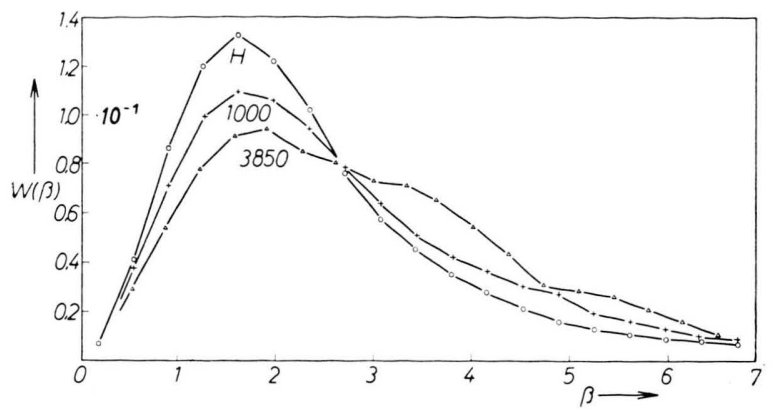

Abb. 1. Mikrofeldverteilung in normierter Darstellung. $\beta=E / 2,61 \cdot e n^{2 / s} ; O=$ Holtsmark-Verteilung $(\mathrm{H})$ für Teilchenzahl $N=\infty$, integriert in Kanalbreiten $\Delta \beta=0,363$; $+=$ „beobachtete“ Verteilung für $N=1000, \Delta=$ desgl. für $N=3850$.

Anm. b. d. Korr.: Die Kurve für $N=3850$ ist mit Fortführung der Integration inzwischen weiter geglättet worden. Das Maximum liegt bei $\beta \approx 2,3$.

Länge größer als mittlerer Teilchenabstand) hergeleitet worden ist, wo sich auf Grund des weitreichenden Couцомв-Feldes ferner Ladungen eine Gauss-Verteilung ergeben sollte. Der durchgeführte Versuch kann mit seinen geradlinigen Bahnen als repräsentativ angesehen werden, da die Teilchenbahnen in Wirklichkeit unter den angegebenen Bedingungen innerhalb von Dimensionen einer Deвye-Länge und noch weit darüber hinaus bekanntlich nahezu geradlinig verlaufen; die im idealen Gas fehlende Wechselwirkung stellt daher keinen Einwand gegen das Versuchsergebnis dar. Für die Feststellung der beobachteten Abweichungen von der HoutsMARK-Verteilung erwies sich bei den der Untersuchung zugänglichen Teilchenzahlen $N$ als wichtig, daß das

1 J. Holtsmark, Ann. Phys., Lpz. 58, 577 [1919].

2 K. Hunger u. R. W. Larenz, Z. Phys. 163, 245 [1961].

3 K. Hunger u. R. W. Larenz, Beiträge aus d. Plasmaphysik 3,161 [1963].
Plasma eine Geschwindigkeitsverteilung aufweist; die thermischen Schwankungserscheinungen sind demnach wesentlich. Für den Gravitationsfall (Ladungen eines Vorzeichens) wurde die gleiche Feldverteilung beobachtet, wie nicht anders zu erwarten.

Die beobachteten deutlichen Abweichungen von der Holtsmark-Verteilung als bekanntlich derjenigen Verteilung, die wesentlich nur den Einfluß des „nächsten Nachbarn" wiedergibt, lassen sich daraus erklären, daß in dem ursprünglichen Holtsmarkschen Ansatz selbstverständlich auch ein Fernfeldanteil enthalten ist, dieser aber bei der dort gewählten Art des Grenzübergangs $N \rightarrow \infty$ verloren geht. Wie in einer demnächst erscheinenden Arbeit gezeigt wird, gewinnt das Fernfeld mit steigender Teilchenzahl $N$ zunehmend an Einfluß. Die Fernfeldverteilung ist eine Gauss-Kurve, deren Parameter durch die Plasmawechselwirkung wie in ${ }^{2}$ bestimmt ist. Die vollständige Mikrofeldverteilung entsteht durch Faltung dieser Gaussschen Fernfeldverteilung mit der Holtsmarkschen Nahfeldverteilung, was jedoch nur für $k T \leqq e^{2} n^{1 / 3}$ von Bedeutung ist. Auf die Wirkung ferner Massen im Gravitationsfall ist bereits von NeumanN ${ }^{4} 1896$ und Seeliger ${ }^{5} 1895$ hingewiesen worden.

Zur Prüfung der Frage, welchem Feld ein (bewegtes) Plasmateilchen ausgesetzt ist, wurden in einer zweiten Gruppe von Versuchen bei kleinerer Teilchenzahl $N \leqq 96$ in Anlehnung an Rechnungen von v. Hoerner ${ }^{6}$ - hier aber unter der Voraussetzung $k T>e^{2} n^{1 / 3}-$ die Teilchenbahnen unter dem Einfluß gegenseitiger Wechselwirkung aller Teilchen berechnet und die Wahrscheinlichkeitsverteilung der auf herausgegriffene Testteilchen wirkenden Feldstärke ermittelt. Bei dieser kleinen Zahl $N$ ergab sich, wie bereits gesagt, eine nur unwesentlich von der Holtsmark-Verteilung abweichende Funktion, und zwar unabhängig davon, ob die Feldstärke am Orte eines Teilchens oder an einem unbesetzten Aufpunkt registriert wurde. Diese Feststellung ist ohne weiteres verständlich, wenn die Feldwirkung weitgehend als kollektive Wirkung entfernterer Teilchen aufgefaßt werden kann (vgl. hierzu auch Ergebnisse von Miller ${ }^{7}$ ).

4 C. Neumans, Über das Newtonsche Prinzip der Fernwirkung, Verlag Teubner, Leipzig 1896.

5 H. Seeliger, Astronom. Nachr. 137, 129 [1895].

6 S. v. Hoerner, Z. Astrophys. 50, 184 [1960]; 57, 47 [1963].

7 R. H. Miller, Astrophys. J. 140, 250 [1964]. 